\title{
SIND GESETZE (NOCH IMMER) ZU HOCH AUFGEHÄNGT?
}

Es braucht nicht besonders betont zu werden, dass Gesetze für den fachlichen Laien eine rätselhafte und fast unverständliche Lektüre darstellen. Im vorliegenden Beitrag wird versucht, deutlich zu machen, warum - bildlich gesprochen - Gesetze zu hoch aufgehängt sind; oder mit anderen Worten: worauf die meisten Verständnisschwierigkeiten von Gesetzestexten zurückzuführen sind. Wurzelt die Unverständlichkeit von Gesetzen in ihrer sprachlichen Beschaffenheit (die Stilthese) oder in der mangelnden Referenz auf die betreffenden Bezugssysteme (die Wissensthese)?

\section{Einleitung}

Die einleitende Diskussion sei mit einem Zitat Els Oksaars $(1988,185)$ eröffnet:

Vor mehr als zweitausend Jahren liess der Tyrann Dionysios von Syrakus die Gesetze so hoch aufhängen, dass sie kein Bürger lesen konnte. Hegel vergleicht in seiner Rechtsphilosophie dieses Unrecht mit der Tatsache, dass das Recht seiner Gestalt nach nur denen zugänglich gemacht wird, die sich - wie er es ausdrückt - gelehrt darauf legen. Heute kann jeder Bürger, wenn er will, alle Gesetze lesen, es scheint jedoch, dass sich seit Dionysios im Prinzip nicht viel geändert hat: sind nicht Gesetze auch heute noch zu hoch aufgehängt, und zwar auf der Abstraktions- und Begriffsleiter der Sprache?

Diese - wohl rhetorische - Frage kann ohne langes Überlegen bejaht werden. Nicht selten wird über die Unverständlichkeit von Gesetzen, über ihre abstrakten Formulierungen und hermetischen Konstruktionen diskutiert, sowohl in Laien- wie auch in Fachkreisen. Die ,sprachlichen Mängel“ der (deutschen) Gesetzessprache sind zum Stoff einer Vielzahl sprachwissenschaftlicher Abhandlungen gemacht worden, ,von allen diesen wissenschaftlichen Bemühungen ist jedoch relativ wenig zur Förderung des Laienverständnisses ausgegangen“ (Warnke 1996, 211).

Wenn uns Beispiel 1 unten überzeugen kann, dass eine ,bürgernahe Gesetzessprache“, die „gefällig - gekürzt - genau - geordnet" (Grosse 1983, 104) ist, möglich und zweifellos auch sehr willkommen wäre und sich die Unverständlichkeit allein durch Stilverbesserung beseitigen ließe, so muss diese Annahme beim Beispiel 2 ( $\S 164,2$ BGB) wieder in Frage gestellt werden:

1 Der vorliegende Beitrag stellt einen Ausschnitt aus meiner Dissertation mit dem Titel "Formale und semantische Strukturen in der deutschen Rechtssprache" dar. Die Arbeit ist von Prof. Dr. Siegfried Heusinger (Mentor, 1992-1996 Gastprofessor and der Universität Ljubljana) und Prof. Dr. Janez Kranjc (Komentor, Juristische Fakultät in Ljubljana) betreut worden. Die Verteidigung der Dissertation fand am 13. Juli 1999 statt (die Kommission: Prof. Dr. Siegfried Heusinger, Prof. Dr. Anton Janko, Prof. Dr. Janez Kranjc). 
Beispiel 1:

statt so:

Der am 20. März 1980 eingegangene Antrag auf Einleitung eines Verfahrens zur Erteilung der Erlaubnis zum Führen eines Kraftfahrzeuges auf öffentlichen Straßen ...

besser so:

Der am 20. März 1980 eingegangene Führerscheinantrag ... (Bürgernahe Sprache 1981, 20, zitiert in: Grosse 1983, 105).

\section{Beispiel 2: § $164 \mathrm{BGB}^{2}$}

1. Eine Willenserklärung, die jemand innerhalb der ihm zustehenden Vertretungsmacht im Namen des Vertretenen abgibt, wirkt unmittelbar für und gegen den Vertretenen. Es macht keinen Unterschied, ob die Erklärung ausdrücklich im Namen des Vertretenen erfolgt oder ob die Umstände ergeben, dass sie in dessen Namen erfolgen soll.

2. Tritt der Wille, im fremden Namen zu handeln, nicht erkennbar hervor, so kommt der Mangel des Willens, im eigenen Namen zu handeln nicht in Betracht $^{3}$.

Trotz einer relativ einfachen syntaktischen Struktur und (scheinbar) verständlichen Lexik bereitet der Absatz 2 des $\S 164$ BGB dem Laien erhebliche Verständnisschwierigkeiten, die nicht auf die „stilwidrige“ sprachliche Enkodierung zurückzuführen sind. Für die Förderung des Laienverständnisses wäre eine paraphrasierte, fallbezogene Erklärung (vgl. Fußnote 3) zweifellos angebracht. Andererseits ließe sich diese Strategie in der Praxis kaum bzw. nicht verwirklichen. Zum einen nähmen fallbezogene Erklärungen wesentlich mehr Platz in Anspruch, andererseits ist die abstrakte Formulierungsweise des Gesetzestextes unentbehrlich: das Recht findet stets auf das soziale Leben Anwendung, das nicht nur eine Unmenge sich voneinander unterscheidender Einzelfälle enthält, sondern zugleich einem ständigen Wechsel unterworfen ist. Im Prozess der Gesetzesanwendung wird versucht, einen konkreten Sachverhalt einer Rechtsnorm unterzuordnen, auf diese Weise zu einer entsprechenden Rechtsfolge zu gelangen, um sie daraufhin auf den konkreten Sachverhalt anwenden zu können. Je abstrakter die Rechtsnorm kodiert, desto einfacher (der Regel nach) der Subsumtionsprozess.

Um die obigen Überlegungen kurz zusammenzufassen: Das Problem der Unverständlichkeit der Rechtssprache - dabei insbesondere der Gesetzessprache - ist viel-

$2 \mathrm{BGB}=$ das Bürgerliche Gesetzbuch aus dem Jahre 1896, das die wesentlichen Regelungen auf dem Gebiet des deutschen Zivilrechts enthält.

3 Eine fallbezogene Erklärung: "Im zitierten zweiten Absatz wird klargestellt, dass der Vertreter unter Umständen selbst die Folgen zu tragen hat, wenn nicht deutlich wird, dass er im Auftrag, in Vertretung handelt. Mit anderen Worten: wenn A im Auftrag von B ein Auto kaufen will, dann kann A im schlimmsten Fall passieren - nämlich dann, wenn der Verkäufer nicht wusste, dass $A$ das Auto nicht für sich, sondern für $B$ kaufen wollte, und B sich plötzlich anders besinnt und kein Auto mehr haben will -, dass er, A, das Auto selbst bezahlen muss" (Joisten 1985, 52). 
schichtig und lässt sich bei weitem nicht nur auf die sprachliche Unverständlichkeit auf die formale Ebene - beschränken.

Im vorliegenden Beitrag versuche ich deutlich zu machen, worin die Unverständlichkeit von Gesetzestexten wurzelt. Da die Spezifika des Gesetzestextes gerade durch den Vergleich zu anderen juristischen Textsorten am besten zum Tragen kommen, wurden neben Gesetzestexten auch einige andere Rechtstexte analysiert (im Rahmen dieses Beitrags werden nur einige Charakteristika dieser Texte erwähnt; Genaueres hierzu vgl. Gruntar Jermol 1999, Kapitel 6). Das untersuchte Korpus umfasst Folgendes: Gesetz (genauer das BGB), Kommentar (zum BGB), juristisches Lehrbuch, Vertrag, Gerichtsurteil und populär-juristischer Text. Um zu möglichst genauen Ergebnissen kommen zu können, wurden bei allen sechs Untersuchungsquellen die (Teil-)Texte ausgewählt, die sich thematisch decken. Die Analyse ist nach folgenden Kriterien durchgeführt worden: Situationstyp, Funktion, thematische Entfaltung, Lexik, grammatische Kennzeichen und Abstraktionsgrad.

\section{Der Situationstyp}

Die Gesetzmäßigkeiten der Situation, in der der Gesetzestext zustandekommt, bestimmen seine Funktion und seine textinternen Komponenten. Da diese Eigenschaften im Folgenden genauer behandelt werden sollen, wird an dieser Stelle der Situationstyp nur in seinen wesentlichsten Zügen geschildert.

Das Gesetz ist eine Sammlung von Rechtsnormen; diese regeln einen bestimmten Lebensbereich, in dem Menschen personelle und sachliche Beziehungen eingehen; sie sind als solche verbindlich und sollen im sozialen Verkehr beachtet werden. Von allen untersuchten Texten ist das Gesetz der abstrakteste. Diese Eigenschaft lässt sich folgendermaßen erklären: Beim Gesetz geht es um die Kodifikation von Rechtssätzen. Soll man diese in der Rechtspraxis auf konkrete Fälle anwenden, so müssen sie möglichst allgemein, d.h. ausreichend abstrakt konzipiert sein. Da einzelne Rechtsnormen einer Erläuterung bzw. Einschränkung bedürfen oder da bestimmte Begriffe schon anderswo (im Rahmen anderer Rechtsnormen) formuliert und definiert sind, findet man im Gesetzestext zahlreiche implizite und explizite Verweise (mehr hierzu unter Punkt 4). Bei diesen handelt es sich nicht selten um Intertextualität; d.h. Verweise beziehen sich auf andere Rechtsquellen (andere Gesetze), wodurch unnötige Wiederholungen vermieden werden und so eine ökonomische Ausdrucksweise erreicht wird. Andererseits hat man es mit einem Text zu tun, der aufgrund zahlreicher Verweise, „mangelnder" Textkohärenz und seiner anspruchsvollen sprachlichen Formulierung abstrakt und schwer verständlich wirkt. Dass aber die abstrakte Formulierungsweise im Gesetzestext unerlässlich ist, wurde am Anfang dieses Beitrags schon betont.

Gesetze werden von Juristen mit der Intention verfasst, einen gewissen Lebensbereich der Bürger (so zumindest im Zivilrecht) rechtlich zu regeln. Dass solche anspruchsvollen Texte den Bürgern unzugänglich sind, steht außer Zweifel. Der Bürger ist fachlicher Laie und besitzt dementsprechend weder das theoretische Fachwissen 
noch praktische Erfahrungen, die Verfasser von Gesetzen einfach als bekannt voraussetzen.

\section{Die Funktion}

Die Textfunktion wird "von außen" - aus der Konstellation der Situationsfaktoren (dem Kommunikationsbereich, den Kommunikationspartnern, ihrer Beziehung zueinander und der kommunikativen Absicht) bestimmt.

Unterschiedlichen Texten liegen genauso unterschiedliche Textfunktionen zugrunde. Einige Texte - wie beispielsweise der Gesetzestext - legen allgemeine Regeln fest, die dann auf konkret-individuelle Situationen angewendet werden. Andere (wie etwa der Vertrag, das Gerichtsurteil und der populär-juristische Text) wiederum gehen von konkreten Fällen aus und versuchen, sie über die abstrakt-generellen Rechtsnormen einzuordnen.

Wenn man die im Rahmen der thematischen Entfaltung dargestellten Arten von Rechtssätzen mitberücksichtigt, kann man feststellen, dass neben den erläuternden, einschränkenden und verweisenden unvollständigen Rechtssätzen die vollständigen bestimmenden im Vordergrund stehen. Sie bestimmen das Handeln und geben an, welche Rechtsfolgen im Falle des Nichtbeachtens oder Zuwiderhandelns zu erwarten sind. Der Gesetzestext drückt eine direkte Aufforderung zum Handeln oder zum Unterlassen des Handelns auf, definiert Handlungsspielräume und bestimmt innerhalb dieser mögliche Handlungsschritte, schreibt vor - gebietet oder verbietet - und begrenzt freie Entscheidungen. Solche Texte "dienen der Sicherung sozialer, institutionsgebundener Handlungen" (Möhn/Pelka 1984, 7). Es besteht ein institutionell geregeltes Abhängigkeitsverhältnis zwischen einer Institution, die befugt ist vorzuschreiben, und den Handelnden, die die gesetzten Handlungsspielräume nicht überschreiten dürfen.

In diesem Sinne kann man von der direktiven oder normierenden Funktion des Gesetzestextes sprechen (vgl. hierzu Möhn/Pelka 1984, 4 ff.). Direktive Texte (das Gesetz) regeln, u.z. in verbindlicher Form. Die Verbindlichkeit äußert sich unter anderem insbesondere in den Textteilen, die auf Rechtsfolgen (manchmal Sanktionen) im Falle der Nichtbeachtung der Bestimmungen oder des Zuwiderhandelns gegen sie hindeuten:

Der Vollmachtgeber kann die Vollmachtsurkunde durch eine öffentliche Bekanntmachung für kraftlos erklären; die Kraftloserklärung muss nach den für die öffentliche Zustellung einer Ladung geltenden Vorschriften der Zivilprozessordnung veröffentlicht werden... ( $\$ 176,1$ BGB).

Das hohe $\mathrm{Ma}$ an Verbindlichkeit bedingt ein ebenso hohes Maß an Genauigkeit und Eindeutigkeit. Dies äußert sich nicht nur auf der lexikalen und syntaktischen Ebene, sondern auch makrostrukturell; der Gesetzestext ist entsprechend den einzelnen Bestimmungen deutlich gegliedert. Als Gliederungssignale fungieren Paragraphen- 
zeichen mit Ziffern und Paragraphenüberschriften. Diese ermöglichen die häufig erforderliche Bezugnahme oder den Verweis auf einzelne Rechtsnormen.

Wie sich das Direktive des Gesetzes auf die sprachliche Ebene niederschlägt, wird im Folgenden genauer dargestellt.

\section{Die thematische Entfaltung}

Unter dem Begriff "thematische Entfaltung" versteht man die gedankliche Ausführung des Themas (vgl. Brinker 1988, 56). Die Möglichkeiten der Entfaltung eines Themas sind verschieden, jeweils gesteuert durch die kommunikative Situation: die Partner, ihre Beziehung, die kommunikative Absicht usw. "Die Entfaltung des Themas zum Gesamtinhalt des Texes kann als Verknüpfung bzw. Kombination relationaler, logisch-semantisch definierter Kategorien beschrieben werden, welche die internen Beziehungen der in den einzelnen Textteilen (Überschrift, Abschnitten, Sätzen usw.) ausgedrückten Teilinhalte bzw. Teilthemen zum thematischen Kern des Textes (dem Textthema) angeben..." (Brinker 1988, 56). Die thematische Entfaltung (Folge von Propositionen) sichert - unter anderem - die semantische Kohärenz des Textes.

Da man bei der thematischen Entfaltung - sei es bei theoretischen Überlegungen oder bei Untersuchungen - die Begriffe Textkohärenz und Textkohäsion nicht umgehen kann, sollen sie an dieser Stelle etwas genauer dargelegt werden.

Als Text kann ein Sprachvorkommnis beschrieben werden, das in sich kohärent ist - d.h. dessen Sätze in irgendeiner Weise miteinander verbunden sind. Die Kohärenz gilt somit als eines der wichtigsten Kriterien der Textualität. Doch wird der Begriff lediglich als eine Eigenschaft von Texten verstanden - nur auf die ausdrucksseitig explizit formulierte Satzverknüpfung beschränkt - so ist er zu eng gefasst. In der neueren (pragmatisch orientierten) Textlinguistik ist weitgehend die Meinung vertreten, dass die Kohärenz erst durch den Empfänger hergestellt werden kann: „Ob einem sprachlichen Gebilde Kohärenz zugesprochen wird, ist nicht nur durch seine Struktur bedingt, sondern auch von der Verstehens- bzw. Interpretationskompetenz des Rezipienten abhängig" (Brinker 1988, 11). Die Kohärenz ist so primär ein semantisches Phänomen, das sich nur begrenzt durch ausdrucksseitig beschreibbare Erscheinungen manifestiert. Aus diesem Grunde hat man in der Sprachwissenschaft den Begriff „Kohäsion“ eingeführt:

Kohäsion betrifft die Art, wie die Komponenten des Oberflächentextes, d.h. die Worte, wie wir sie tatsächlich hören und sehen, miteinander verbunden sind. Die Oberflächen-komponenten hängen durch grammatische Formen und Konventionen voneinander ab, so dass also Kohäsion auf grammatischen Abhängigkeiten beruht. Kohärenz betrifft die Funktionen, durch die die Komponenten der Textwelt, d.h. die Konstellationen von Konzepten (Begriffen) und Relationen (Beziehungen), welche dem Oberflächentext zugrunde liegen, für einander gegenseitig zugänglich und relevant sind (de Beaugrande/Dressler 1981, 3 f.). 
Die Abgrenzung von Kohäsion und Kohärenz ist oft auf Kritik gestoßen:

Eine separate Behandlung von Kohäsion und Kohärenz ist nicht folgerichtig. Wenn wir auch Kohäsion und Kohärenz zunächst getrennt betrachten, so wird sich doch ... zeigen, dass der Kohäsionsbegriff zu einem Großteil in den interdisziplinär ausgerichteten Kohärenzbegriff integriert werden muss (van de Velde 1981, 27, zitiert in: Busse 1992, 45).

Das Problem der Abgrenzung der semantisch und pragmatisch begründeten Kohärenz von der grammatisch aufgefassten Kohäsion liegt schon in der Tatsache, dass sprachliche Zeichen eine untrennbare Einheit von Ausdrucks- und Inhaltsseite darstellen. In dieser bilateralen Sicht werden durch sprachliche Zeichen sowohl formale wie auch inhaltliche Beziehungen vermittelt. Daraus folgt, dass auch eine analytische Trennung von grammatikalischer und inhaltlicher bzw. thematischer Seite des Textes nicht möglich ist, denn in konkreten sprachlichen Äußerungsakten bedingen sich die beiden Gebiete gegenseitig (vgl. Busse 1992, 46).

Bei der näheren Betrachtung der grammatischen Verknüpfungssignale (in der Sprachwissenschaft gibt es dafür folgende Termini: Ko-Referenz, Substitution, Pronominalisierung, Verweisung, Wiederaufnahme ...) stellt sich bald heraus, dass sich auch diese auf die Grundeigenschaft von Textkohärenz beziehen. Denn bei (fast) jeder Form von Bezügen zwischen Satzteilen und Sätzen handelt es sich um eine semantische Verknüpfung, „weil auch die sogenannten 'grammatischen' Verknüpfungssignale ihre volle Wirkung nur über die Kenntnis der ganzen Satzinhalte entfalten können “ (Busse 1992, 44). Die rein oder vorwiegend grammatischen Verknüpfungssignale (so wie etwa transitive Verben, die das Akkusativobjekt verlangen, oder Präpositionen ihren Kasus) sind selten. Kann man die Rekkurenz ${ }^{4}$ (direkte Wiederholung von Elementen) noch als rein grammatische Verknüpfung verstehen, so ist dies beim Parallelismus (Wiederholung syntaktischer Oberflächenstrukturen, die aber mit verschiedenen Ausdrücken ausgefüllt sind), bei der Paraphrase (der Rekurrenz des Inhalts mit einer Änderung des Ausdrucks) sowie bei der Junktion (dient dem Ausdruck kausaler, konditionaler u.a. Beziehungen) nicht ohne weiteres anzunehmen. Dabei handelt es sich nämlich schon um semantisch-strukturelle Beziehungen.

Vom Allgemeinen nun zum Konkreten: Um feststellen zu können, ob und wie die kleinsten optisch markierten Einheiten des Gesetzestextes - die Paragraphen - semantisch miteinander verbunden sind, wurden die $\S \S 164-181$ BGB untersucht. Diese befinden sich unter der Überschrift "Fünfter Titel: Vertretung. Vollmacht". Der fünfte Titel ist eingeordnet in eine systematische Gliederung des Gesamttextes des BGB, u.z. in einen der fünf bzw. sechs Großabschnitte mit dem Titel "Allgemeiner Teil" (Erstes Buch). Dieser Großabschnitt teilt sich dann in mehrere kleinere Abschnitte, diese wiederum in einzelne Titel. Jeder Titel besteht aus einer Reihe von Paragraphen oder

4 Zu Rekurrenz, Parallelismus, Paraphrase, Junktion u.a. vgl. de Beaugrande/Dressler 1981, $50 \mathrm{ff}$. 
Artikeln. Paragraphen sind fortlaufend nummerierte kleine Abschnitte eines Gesetzes, in denen einzelne Rechtsnormen (d.h. Rechtssätze) festgelegt werden.

Bei den Untersuchungen wurde nach den semantischen Beziehungen zwischen den einzelnen Paragraphen gesucht. Man wollte nämlich feststellen, ob sich aus dem Aneinanderreihen der Paragraphen eine thematische Entfaltung ergibt. Innerhalb der einzelnen Paragraphen sind keine genaueren derartigen Untersuchungen durchgeführt worden, so dass mir hierzu auch keine eingehenden Belege zur Verfügung stehen. Trotzdem kann angenommen werden, dass der Paragraph als ein kohärenter Teiltext zu betrachten ist - schon deshalb, weil er die Festlegung einer Rechtsnorm zum Inhalt hat.

Aus der Analyse der $\S \S 164-181$ BGB geht hervor, dass die Rechtsnormen innerhalb des Titels "Vertretung. Vollmacht" zwar thematisch zusammenfallen, dass sie aber trotzdem als relativ selbständige Einheiten auftreten, so dass von einer thematischen Entfaltung und daher von einem kohärenten Text kaum die Rede sein kann. Auch wenn die aneinandergereihten Paragraphen dieselbe Bezugsgröße (Referenz) haben, wie z.B. in den $\S \S 177-180$ BGB, wo „das Handeln ohne Vertretungsmacht“ bestimmt ist, werden die Einheiten (Paragraphen) weder durch formale Mittel (durch das System syntaktischer Mittel) noch inhaltlich miteinander verbunden. In diesem Sinne erscheinen die einzelnen Paragraphen als mehr oder weniger selbständige Teiltexte, was durch das Beispiel unten zusätzlich bewiesen wird.

Der unbestimmte Artikel mit seiner kataphorischen (vorausweisenden) und der bestimmte Artikel mit der anaphorischen Funktion sind typische Mittel zur Herstellung der Textkohäsion. Da aber die Textkohäsion wiederum die Grundlage der semantischen Kohärenz darstellt, wirken dementsprechend auch der unbestimmte und bestimmte Artikel als kohärenzbildende Elemente.

Im § 241 BGB (Zweites Buch: Recht der Schuldverhältnisse. Erster Abschnitt. Erster Titel: "Verpflichtung zur Leistung") wird der Inhalt des Schuldverhältnisses bestimmt:

Kraft des Schuldverhältnisses ist der Gläubiger berechtigt, von dem Schuldner eine Leistung zu fordern. Die Leistung kann auch in einem Unterlassen bestehen.

Die Bezugsgrößen "Gläubiger" und "Schuldner" finden sich gleich am Anfang des zweiten Buches des BGB, eingeleitet mit einem bestimmten Artikel. Für die beiden gibt es im Vortext (Erstes Buch: Allgemeiner Teil) keine Bezugsstellen - nicht einmal im Rahmen des dritten Titels "Vertrag" (dritter Abschnitt). Vielleicht liegt die Erklärung dafür in der spezifischen Art des Formulierens von Gesetzestexten. Denn zu einem Gesetzesparagraphen greift man dann, "wenn der Bezug auf eine Sache ... durch den zu entscheidenden Fall kontextuell bzw. situativ schon vorliegt" (Busse 1992, 63). Andererseits wird durch das obige Beispiel die Behauptung bestätigt, dass der Paragraph sich durch einen relativ selbständigen Charakter auszeichnet.

$\mathrm{Da}$ aber das Gesetz trotzdem als ein selbständiger Text (und auch als eine Textsorte) betrachtet werden kann (vgl. hierzu Busse 1992, 73 ff.), muss er als solcher neben den 
anderen Textualitätskriterien (vgl. de Beaugrande/Dressler 1981) auch das Kriterium "Textkohärenz" erfüllen.

Wie schon erwähnt, besteht ein Gesetz in der Regel aus einer Vielzahl von Rechtssätzen. Rechtssätze stellen Verhaltensnormen für den Bürger und zugleich Entscheidungsnormen für die Gerichte und die Behörden dar. Sie haben einen normativen und generellen Charakter; Letzteres bedeutet, dass Rechtssätze "nicht nur gerade für einen bestimmten Fall, sondern innerhalb ihres räumlichen und zeitlichen Geltungsbereichs für alle Fälle 'solcher Art"" gelten (Canaris/Larenz 1995, 71). Oder in anderen Worten: Sie lassen sich auf ähnliche Fälle anwenden und müssen als solche abstrakt formuliert sein.

Im Folgenden beschränke ich mich nicht mehr nur auf die $\S \S 164-181$ BGB, da für derartige Untersuchungen ein breiterer Kontext erforderlich ist.

Der Rechtssatz ${ }^{5}$ hat die sprachliche Form eines Satzes:

Nach dem Erlöschen der Vollmacht hat der Bevollmächtigte die Vollmachtsurkunde dem Vollmachtgeber zurückzugeben; ein Zurückbehaltungrecht steht ihm nicht zu (§ $175 \mathrm{BGB})$.

Dieser Satz schreibt allen, die im Sinne dieser Bestimmung als Bevollmächtigte anzusehen sind, ein bestimmtes Verhalten vor. Er verbindet einen generell umschriebenen Sachverhalt - einen Tatbestand - mit einer Rechtsfolge, d.h. der Rechtssatz ordnet einem generellen Tatbestand eine Rechtsfolge zu (vgl. Canaris/Larenz 1995, 72). Beim obigen Beispiel ist die Rechtsfolge die Verpflichtung des Bevollmächtigten zur Rückgabe der Vollmachtsurkunde. Sprachlich wird das kenntlich gemacht durch die Form "hat $+z u+$ Infinitiv" (hat ... zurückzugeben). Dies kann paraphrasiert werden durch "soll + Infinitiv" (soll ... zurückgeben) oder "ist verpflichtet $+\mathrm{zu}+$ Infinitiv" (ist verpflichtet ... zurückzugeben). Alle drei Formen sind charakteristisch für die Sprache des Normativen, denn die "Rechtsfolge liegt stets auf dem Gebiet des Normativen" (Canaris/Larenz 1995, 72).

Rechtssätze sind Bestimmungssätze. Sie erscheinen in der Form von "Sollsätzen". Da nicht jeder Rechtssatz unbedingt ein Gebot oder Verbot zum Ausdruck bringt, wohl aber eine Geltungsanordnung, können Rechtssätze nicht als Imperative bzw. Befehle verstanden werden, sondern als Bestimmungen ${ }^{6}$. Die Bestimmung impliziert, dass etwas sein soll, dass das Bestimmte maßgeblich ist.

Nicht alle Rechtssätze sind vollständig. Einige haben lediglich die Funktion, den Tatbestand oder die Rechtsfolge genauer zu bestimmen, einzuschränken oder auf einen

5 Jeder vollständige Rechtssatz setzt sich aus Tatbestand und Rechtsfolge zusammen. Der Tatbestand ist etwas Abstraktes, genauer eine abstrakte Situationsbeschreibung. Im Prozess der Subsumtion (= der Vorgang der Rechtsanwendung) wird ein konkreter Lebensvorgang (etwas Einmaliges, ein Lebenskonkretum, das sich im sozialen Leben ereignet hat, auch Sachverhalt genannt) dem abstrakten Tatbestand untergeordnet, u.z. mit der Absicht, für das Konkrete jene Rechtsfolge herbeizuführen, die im Abstrakten bestimmt ist.

6 Zum Unterschied zwischen Bestimmung und Befehl vgl. Canaris/Larenz 1995, 74 ff. 
anderen Rechtssatz zu verweisen. Als Sätze sind sie vollständig, als Rechtssätze nicht. Man spricht daher von unvollständigen Rechtssätzen, die erläuternd, einschränkend und verweisend sein können.

Unvollständige Rechtssätze sind für Untersuchungen zur Textkohärenz besonders wichtig, da sie als eine Art semantischer Verknüpfungen betrachtet werden können, die einzelne Rechtssätze miteinander verbinden. Das erfolgt nicht nur innerhalb eines thematischen Zusammenhangs (z.B. eines Titels), sondern geht über seine Grenzen hinaus.

Durch erläuternde Rechtssätze werden Tatbestände oder Rechtsfolgen näher umschrieben:

Fahrlässig handelt, wer die im Verkehr erforderliche Sorgfalt außer acht lässt $(\S 276,1$, Satz 2, BGB) $\rightarrow$ Umschreibung des Begriffs "Fahrlässigkeit".

$\S 433$ BGB z.B. leitet die Regelungen des Vertragstypus ein und erläutert (umschreibt), was das Gesetz unter einem Kaufvertrag versteht, d. h. die Verpflichtungen des Verkäufers und des Käufers. Dass und wie "diese Verpflichtungen von der Rechtsordnung sanktioniert werden, ergibt sich aus den allgemeinen Vorschriften über Verträge" ( $\S ~ 305-327$ BGB, Begründung. Inhalt des Vertrags./[?] Gegenseitiger Vertrag.) "und nicht erst aus $\S 433$ BGB" (Canaris/Larenz 1995, 79). Solche inhaltlichen Verbindungen zwischen Rechtssätzen sind nicht immer explizit verbalisiert; bei vielen handelt es sich eher um implizit formulierte Verweisungen.

Einschränkende Rechtssätze schränken einen vorausgegangenen, weit gefassten Rechtssatz ein, indem sie eine negative Geltungsanordnung angeben ("gilt nicht"). Auch hier - wie schon bei den erläuternden Beispielen oben - zeigt sich die eng verwobene und äußerst komplizierte inhaltliche Struktur des Gesetzestextes. Rechtssätze stehen nicht isoliert nebeneinander, sondern geben eine zusätzliche Erklärung, sie schränken ein oder verweisen auf andere Rechtsnormen, wodurch ein dichtes semantisches Netz entsteht.

Durch $\S \S 399$ und 400 wird der Satz $\S 398$ BGB eingeschränkt:

Eine Forderung kann von dem Gläubiger durch Vertrag mit einem anderen auf diesen übertragen werden (Abtretung)... (§ 398 BGB).

Eine Forderung kann nicht abgetreten werden, wenn die Leistung an einen anderen als den ursprünglichen Gläubiger nicht ohne Veränderung ihres Inhalts erfolgen kann oder wenn die Abtretung durch Vereinbarung mit dem Schuldner ausgeschlossen ist ( $\$ 399 \mathrm{BGB}$ ).

Eine Forderung kann nicht abgetreten werden, soweit sie der Pfändung nicht unterworfen ist ( $\S 400 \mathrm{BGB}$ ).

In diesem Zusammenhang gibt es noch wesentlich kompliziertere Ausdrucksformen mit zweifachen negativen Geltungsanordnungen, wobei die zweite Geltungsan- 
ordnung die erste einschränkt (dazu vgl. § 932 und $\S 935$ BGB). Zuletzt sollen noch verweisende Rechtssätze erwähnt werden:

Auf die Pacht mit Ausnahme der Landpacht sind, soweit sich nicht aus den $\S \S 582$ bis $584 \mathrm{~b}$ etwas anderes ergibt, die Vorschriften über die Miete entsprechend anzuwenden ( $(581,2 \mathrm{BGB})$.

Im $\S 581$ wird das Wesen des Pachtvertrags bestimmt. Dabei wird im 2. Absatz auf die Vorschriften über die Miete verwiesen ( $\S \S 535-580$ BGB), die mit gewissen Einschränkungen auf die Pacht anwendbar sind. Verweisende Rechtssätze erscheinen oft in der Form von expliziten Querverweisen, u.z. durch die stereotype Formulierung "findet/n entsprechende Anwendung". Dabei können sich einzelne Verweisungen auf sich weit hinten oder vorne befindende Rechtssätze beziehen (z.B. der $\S 571$ verweist u.a. auf den $\S 771 \mathrm{BGB})$. Zu finden sind außerdem auch Verweise auf Paragraphen anderer Gesetze (außerhalb des BGB):

Wird dem Mieter von Wohnraum nach $\$ 721$ oder 794a der Zivilprozessordnung eine Räumungsfrist gewährt... ( $\$ 557,3 \mathrm{BGB}$ ).

Die Verweisung ist einerseits "ein gesetzestechnisches Mittel, um umständliche Wiederholungen zu vermeiden" (Canaris/Larenz 1995, 82), andererseits erhöht sich dadurch der Schwierigkeitsgrad (denn Texte mit zahlreichen Verweisen erschweren die Rezeption) und dadurch auch der Abstraktionsgrad des Textes. Darüber hinaus sind verweisende Rechtssätze eines der Textkohärenzmerkmale.

Wie aus dem bisher Geschilderten ersichtlich, sind Gesetze in der Regel recht komplizierte und abstrakte Texte. Ihre genaue Bedeutung ist immer wieder problematisch, was auch daran liegt, dass sie "keine in ihrem Umfang genau festgelegten Begriffe verwenden, sondern mehr oder minder flexible Ausdrücke, deren mögliche Bedeutung innerhalb einer weiten Bandbreite schwankt und je nach den Umständen, der Satzbezogenheit und dem Zusammenhang der Rede, der Satzstellung und Betonung eines Wortes unterschiedlich sein kann" (Canaris/Larenz 1995, 133). Das Gesetz definiert einige Begriffe nicht (wie Rechtsgeschäft, subjektives Recht, rechtswidrig), wieder andere - z.B. Fahrlässigkeit - sind unvollständig oder mehrdeutig. Darüber hinaus gibt es für den gleichen Sachverhalt Rechtsfolgen, die einander ausschließen können. Wenn sie sich nicht ausschließen, stellt sich die Frage, ob sie nebeneinander eintreten sollen, oder ob die eine die andere verdrängt (vgl. Canaris/Larenz 1995, 133f.). Daraus ergibt sich die Notwendigkeit der Auslegung von Gesetzestexten - ein Beweis mehr dafür, dass Gesetzestexte von allen juristischen Fachtexten die abstraktesten sind.

7 Gewiss ist aber der juristische Begriff - Terminus - im Vergleich zum gemeinsprachlichen Wort inhaltlich genau festgelegt, d.h. monosem. 


\section{Die lexikalischen Eigenschaften}

Dass der Gesetzestext durch einen hohen Anteil an Termini gekennzeichnet ist, braucht nicht betont zu werden. Im Folgenden konzentriere ich mich nicht auf die Darstellung einzelner Textsorten in Bezug auf ihre lexikalischen Charakteristika, sondern vielmehr auf einen zusammenfassenden Vergleich der untersuchten Texte bzw. auf die Ergebnisse, die sich bei einer kontrastiven Analyse ergeben haben.

Obwohl die Untersuchungen im Großen und Ganzen auf den terminologischen Kern im jeweiligen Text beschränkt sind, möchte ich an dieser Stelle nur kurz auf die lexikalischen Mittel verweisen, die deutlich den direktiven Charakter der Gesetzessprache zum Ausdruck bringen:

Bestimmung, bestimmen, Regel, Vorschrift, Erfordernis, (un)zulässig sein, gewährleisten, gestatten, verboten sein, vorgeschrieben sein, gelten für, erforderlich sein, rechtliche Folgen, bestimmen, verpflichtet sein, Pflicht, wirksam sein, Wirkung haben, maßgebend sein, müssen, sollen, können, berechtigt sein, beschränkt sein, Anwendung finden ...

Die Untersuchungen haben ergeben, dass im terminologischen Teil der Lexik die Unterschiede zwischen den analysierten Texten sehr gering sind. So enthalten der Kommentar und das Lehrbuch einige Ausdrücke, die für die Rechtstheorie spezifisch sind, nicht jedoch für die Sprache des Gesetzes. Dabei handelt es sich um den begrifflichen Aufbau (Verallgemeinerung) von gesetzlichen Bestimmungen. Unter diesen Ausdrücken sind nicht selten Termini lateinischer Herkunft $\mathrm{zu}$ finden, oder man bedient sich direkt lateinischer/griechischer Ausdrücke:

Repräsentationstheorie, Offenlegungsgrundsatz, hypothetisches Erfüllungsinteresse, Kulpakompensation, positive Forderungsverletzung, culpa in contrahendo...

Interessant sind die Ergebnisse der Analyse, die an dem populär-juristischen Text "Mein Recht im Alltag" durchgeführt worden ist. Der genannte Text ist das einzige Exemplar des untersuchten Korpus, bei dem die Kommunikationspartner Fachleute (als Textproduzenten) und Laien (als Rezipienten) sind. Sicher ist hier im Vergleich zu den anderen Texten (insbesondere zum Gesetz) der Anteil an Termini - prozentual betrachtet - wesentlich geringer. Wegen der Spezifik des Adressatenkreises wären im populär-juristischen Text eher gemeinsprachliche Synonyme bzw. Umschreibungen ${ }^{8}$ für die Termini zu erwarten.

Die Ergebnisse haben diese Erwartungen widerlegt, denn der Text enthält genau dieselben Termini wie alle anderen Texte. Trotzdem enthält der populär-juristische Text einige adressatenspezifische Besonderheiten. Der für die Fachsprache schlechthin typische Nominalstil wird hier nicht selten durch den Verbalstil abgelöst. Der Letztere

8 Unter "Umschreibung" versteht man z.B. die Erklärung durch die Zerlegung des Terminus in eine Wortgruppe; z.B. Gehilfenverschulden = Verschulden durch den Gehilfen (obwohl auch durch das Auflösen der Konstruktion das Verständnis nicht wesentlich erleichtert wird) und/oder eine "vereinfachte" - populärjuristische Definition. 
ist adressatenfreundlicher: Im Gegensatz zum Nominalstil enthält er keine (oder wesentlich weniger) schwerfälligen Konstruktionen, die den Rezeptionsprozess - insbesondere für den fachlichen Laien - wesentlich beeinträchtigen können. Im Text finden sich folgende Beispiele:

arglistig getäuscht werden (für: arglistige Täuschung), etwas mit rückwirkender Kraft anfechten (für: Anfechtung ex tunc), das Grundstück auflassen und übereignen (für: Auflassung und Übereignung des Grundstücks), etwas notariell beglaubigen lassen (für: notarielle Beglaubigung)...

Dem im Verbalstil ausgedrückten Terminus folgt manchmal später im Text auch der "übliche" Terminus (vgl. oben).

Als ein weiteres adressatenspezifisches Merkmal kann man auch orthografische Besonderheiten betrachten:

Mit-sich-selbst-Kontrahieren (für: Selbstkontrahieren), "In-sich-Geschäft" (für: Insichgeschäft, ev. auch Insich-Geschäft)

Die unübliche Schreibweise wird im Text manchmal durch Anführungszeichen (vgl. das Beispiel oben) kenntlich gemacht, wobei später auch hier der Terminus orthografisch korrekt - wie z.B. im Gesetzestext - ausgeschrieben wird. Diese Abweichung in der Orthografie hat die Funktion, den Terminus präsenter und so einigermaßen verständlicher $\mathrm{zu}$ machen.

Die von Wilhelm Schmidt (1969) vorgeschlagene Gliederung der Fachlexik in Termini, Halbtermini und Fachjargonismen lässt sich in der Rechtssprache wohl nicht anwenden. Obwohl meine Untersuchungen auf den schriftlichen Kommunikationsbereich beschränkt sind, kann angenommen werden, dass sich die mündliche Kommunikation im lexikalischen Bereich nicht wesentlich von der schriftlichen unterscheidet. Das lässt sich mit der Tatsache erklären, dass es im Recht sehr auf eine klare, möglichst eindeutige Ausdrucksweise ankommt. Im Recht geht es um die Regelung des Zusammenlebens von Menschen, deshalb "muss größte Genauigkeit in der Formulierung der Norm angestrebt werden" (Sandrini 1996, 11), eine ebenso große Genauigkeit in der Auslegung der Norm (z.B. im Kommentar) und schließlich auch im Prozess der Subsumtion (Unterordnung eines konkreten Sachverhalts unter den Tatbestand einer Rechtsnorm - wie im Urteil oder im Vertrag). Auch in der Kommunikation zwischen dem Fachmann und dem Laien (z.B. Anwalt : Klient) handelt es sich um einen Fall der Subsumtion. Denn bei der Erklärung eines konkreten Falls (Sachverhalts) kann und muss sich der Fachmann des ihm zur Verfügung stehenden Instrumentariums - der kodifizierten Rechtsnormen - bedienen. In diesem Prozess muss der Anwalt ähnlich wie der Richter, der ein Urteil fällt, verfahren: "Im Urteil muss der Richter Entscheidungsgründe angeben; er unterliegt dem sog. Begründungszwang. ... [irgendein Satzzeichen gehört hierher] das läuft darauf hinaus, dass er mitteilt, wie er die Tatbestandsmerkmale der die Entscheidung tragenden Normen versteht (Auslegung) und 
inwiefern er sie den Sachverhaltsmerkmalen als kongruent ansieht (Subsumtion)" (Krawietz 1976, 424, zitiert in: Sandrini 1996, 31).

Aus diesen Tatsachen kann eine logische Schlussfolgerung gezogen werden: Die untersuchten juristischen Fachtexte - inklusive des populär-juristischen Textes - enthalten einen mehr oder weniger festen und identischen terminologischen Kern. Ob und wie einzelne Termini in den einzelnen Texten erklärt werden, ist durch die jeweilige kommunikative Situation bedingt und spielt in diesem Zusammenhang keine Rolle. Gewiss enthält jeder Text (sei es ein Gesetz, ein Vertrag, ein populär-juristischer Text usw.) neben dem terminologischen Kern auch andere lexikalische Elemente, die dem Ausdruck der Intention und des Gegenstandes des juristischen Textes unmittelbar dienen. Auch das unterscheidet ihn von den anderen Fachtexten.

\section{Die grammatischen Kennzeichen}

Der Gesetzestext zeigt eine ausgeprägte Selektion syntaktischer Mittel:

- als Tempus dominiert das Präsens. Für das Vergangene wird das Perfekt, gelegentlich auch das Präteritum gebraucht. Das Präsens verleiht der Aussage eine zeitfreie Gültigkeit;

- während der Konjunktiv selten zu finden ist, erscheint der Imperativ nie. Die Modalitäten der Aussage werden durch die Modalverben können, sollen, müssen und dürfen umschrieben, die in ihrer Graduierung eine eigene rechtssprachliche Semantik haben (vgl. Wagner, 1970, 17, zitiert in: Grosse 1983, 101). Außerdem wird die Modalität durch den modalen Infinitiv ausgedrückt:

...die Kraftloserklärung muss nach den für die öffentliche Zustellung einer Ladung geltenden Vorschriften der Zivilprozessordnung veröffentlicht werden (§ 176, $1 \mathrm{BGB})$.

Die Erteilung der Vollmacht erfolgt durch Erklärung gegenüber dem zu Bevollmächtigenden oder dem Dritten, demgegenüber die Vertretung stattfinden soll (§ 167, 1 BGB).

Der Widerruf kann auch dem Vertreter gegenüber erklärt werden (§ 178 BGB).

Nach dem Erlöschen der Vollmacht hat der Bevollmächtigte die Vollmachtsurkunde dem Vollmachtgeber zurückzugeben ( $\$ 175$ BGB).

Ist an einem bestimmten Tag oder innerhalb einer Frist eine Willenserklärung abzugeben oder eine Leistung zu bewirken... (§ 193).

- obwohl das Passiv auftaucht, herrschen doch Aktivsätze vor, wodurch die Handelnden - die Vertragsparteien - (und nicht der Prozess) in den Mittelpunkt gerückt werden. Es kommt nämlich darauf an, "wer wen womit woran bindet";

- die Bevorzugung von Funktionsverbgefügen, die einfache Verben ersetzen, ist deutlich, z.B.: in Betracht kommen, Anwendung finden, in Kraft bleiben, in Kenntnis setzen.... Das Verb trägt nur noch eine syntaktische Funktionsanzeige - wird "sinnentleert", das Substantiv übernimmt die Bedeutung; 
- eines der spezifischen Merkmale der Sprache des Gesetzes ist auch die unpersönliche Gestaltungsart. Der Bürger wird im Gesetzestext in der dritten Person mit einer substantivischen Umschreibung angeredet. Von Pronomina, die im Vergleich zu Substantiven seltener verwendet werden, sind Indefinit- (jemand, einen anderen, ein anderes...) und gelegentlich auftretende Demonstrativpronomen zu erwähnen. Zur Bezeichnung der handelnden Personen werden oft substantivierte Partizipien eingesetzt (der Vertretene, der Vertretende, der Bevollmächtigte, der Bevollmächtigende).

Wer als Vertreter einen Vertrag geschlossen hat, ist, insofern er nicht seine Vertretungsmacht nachweist, dem anderen Teile nach dessen Wahl zur Erfüllung oder zum Schadensersatze verpflichtet, wenn der Vertretene die Genehmigung des Vertrags verweigert ( $\$ 179,1 \mathrm{BGB}$ ).

- es gibt formelhafte Präpositionalverbindungen, von denen viele substantivierte Verben sind, z.B.: in Zusammenhang mit, zur Erfüllung, zur Vertretung... In diesem Zusammenhang sind oft Partizipien in attributiver Stellung zu finden (eine vor der Aufforderung dem Vertreter gegenüber erklärte Genehmigung, das ihrer Erteilung zugrunde liegende Rechtsverhältnis), einige von ihnen besitzen einen modalen Charakter (eine gegenüber einem anderen abzugebende Willenserklärung);

- Substantive werden mit Hilfe von Genitiv und Präpositionen einander untergeordnet. Auf diese Weise entsteht eine hypotaktische Struktur, deren Sinn schwer nachvollziehbar ist:

nach den<smiles>CC1CC1</smiles>

für die öffentliche Zustellung<smiles>C1CC1</smiles>

einer Ladung

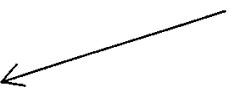

geltenden Vorschriften

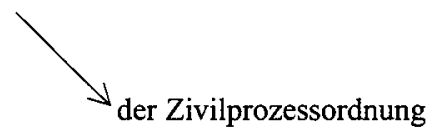

der Erlöschen<smiles>C=IC</smiles>

der Vertretungsmacht

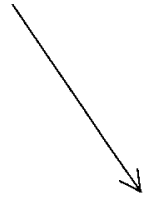

bei der Vornahme<smiles>C1CC1</smiles>

des Rechtsgeschäfts 


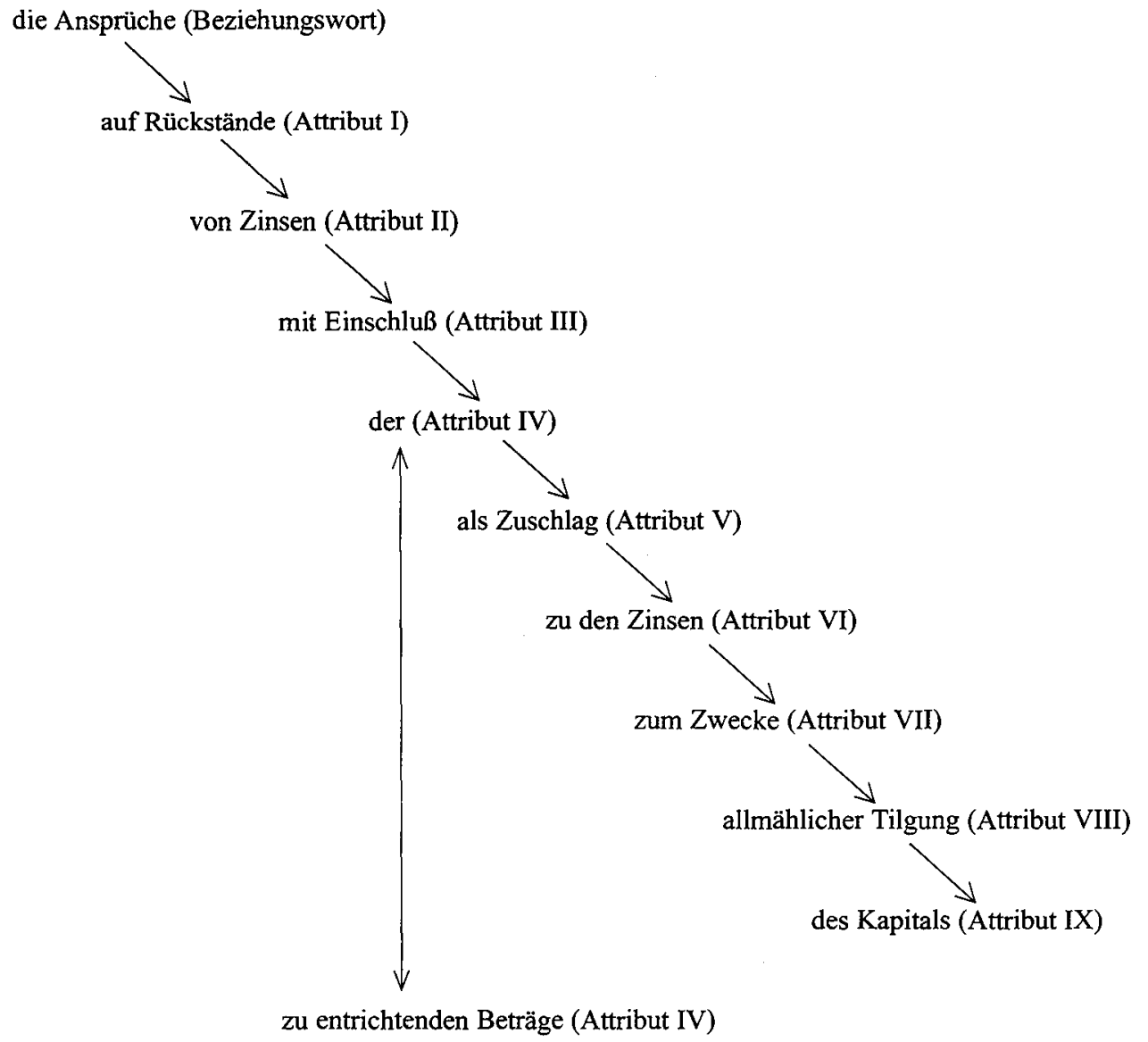

Die obige Struktur findet sich in folgendem Satz (§ 197 BGB):

In vier Jahren verjähren

1. die Ansprüche auf Rückstände von Zinsen, mit Einschluss der als Zuschlag zu den Zinsen zum Zwecke allmählicher Tilgung des Kapitals zu entrichtenden Beträge,

2. die Ansprüche auf Rückstände von Miet- und Pachtzinsen, soweit sie nicht unter die Vorschrift des $\S 196$ Abs. 1 Nr. 6 fallen

3. und die Ansprüche auf Rückstände von Renten, Auszugsleistungen, Besoldungen, Wartegeldern, Ruhegehalten, Unterhaltsbeiträgen und allen anderen regelmäßig wiederkehrenden Leistungen.

Der Satz ist - grob gesehen - einfach strukturiert: Temporalangabe - Prädikat Subjekt. Das Subjekt fügt sich aus einer Wortreihe zusammen, wobei einzelne Teile 
(Subjekte) einmal asyndetisch, das zweite Mal syndetisch miteinander verbunden sind. Jedes Subjekt stellt eine komplexe Struktur dar: Es fängt jeweils mit "die Ansprüche auf Rückstände von" an. Das Beziehungswort (der Gliedkern) "die' Ansprüche" wird mehrfach attribuiert, wobei Attribute bis zum 9. Grad gehen, was zu einer komplizierten hypotaktisch organisierten und deshalb schwer verständlichen Komposition führt (vgl. grafische Abbildung oben). Beim zweiten Teil wird das 2. Attribut durch einen Nebensatz (Modalsatz) eingeschränkt. Beim dritten Teil geht es um eine Aufzählung von Attributen 2. Grades, die vorwiegend asyndetisch miteinander verbunden sind.

- die Satzstruktur des Gesetzes ist relativ kompliziert. Es finden sich Nebensätze unterschiedlicher Art. Dabei fallen häufige uneingeleitete Konditionalsätze ins Auge. Im Konditionalsatz wird der Sachverhalt (Tatbestand) angegeben, im Hauptsatz (eingeleitet durch so) die Rechtsfolge. Die Funktion der Konditionalgefüge ist es, die Bedingungen möglicher oder erwünschter Handlungen präzise und unmissverständlich anzugeben:

Schließt jemand ohne Vertretungsmacht im Namen eines anderen einen Vertrag, so hängt die Wirksamkeit des Vertrags für und gegen den Vertretenen von dessen Genehmigung ab (§ $177 \mathrm{BGB})$.

- Satzgefüge mit mehreren, voneinander abhängigen Nebensätzen sind keine Ausnahme; einige davon können recht lang sein:

In vier Jahren verjähren die Ansprüche auf Rückstände von Zinsen, mit Einschluss der als Zuschlag zu den Zinsen zum Zwecke allmählicher Tilgung des Kapitals zu entrichtenden Beträge, die Ansprüche auf Rückstände von Mietund Pachtzinsen, soweit sie nicht unter die Vorschrift des § 196 Abs. 1 Nr. 6 fallen, und die Ansprüche auf Rückstände von Renten, Auszugsleistungen, Besoldungen, Wartegeldern, Ruhegehalten, Unterhaltsbeiträgen und allen anderen regelmäßig wiederkehrenden Leistungen (§ 197 BGB).

- obwohl die Sprache des Gesetzes die Mitteilung von möglichst viel Informationen in möglichst wenigen Worten anstrebt, leidet[?] sie keine Ellipsen. Auch Proformen sind sehr eingeschränkt verwendet. Das führt zu Wiederholungen - oder genauer zur Wiederaufnahme von Satzgliedern durch Wortwiederholung, was einerseits stilistisch unästhetisch wirkt, aber auf der anderen Seite der Genauigkeit der Aussage dient:

Eine Frist, die nach Wochen, nach Monaten oder nach einem mehrere Monate umfassenden Zeitraume - $\underline{\mathbf{J} a h r}$, halbes $\underline{\mathrm{Jahr}}$, Vierteliahr - bestimmt ist, endigt im Falle des $\S 187$ Abs. 1 mit dem Ablaufe desjenigen Tages der letzten Woche oder des letzten Monats, welcher durch seine Benennung oder seine Zahl dem Tage entspricht, in den das Ereignis oder der Zeitpunkt fällt, im Falle des § 187 Abs. 2 mit dem Ablaufe desjenigen Tages der letzten Woche oder des letzten Monats, welcher dem Tage vorhergeht, der durch seine Benennung oder seine Zahl dem Anfangstage der Frist entspricht ( $\$ 188,2$ BGB). 
Die Häufung von Verbalsubstantiven, die durch Präpositional- und Genitivverbindungen die Simultanität verschiedener Vorgänge/Zustände andeuten, führt zu hypotaktisch gestaffelten Substantivtreppen. Sie erschweren das Verständnis und wirken viel schwerfälliger als die Klammerbildung des finiten Verbs. Darin zeigt sich die deutliche Neigung des Gesetzestextes zum Nominalstil. Dieser bedeutet die Verdichtung der Information, wodurch eine prägnante Ausdrucksweise ermöglicht wird. Die syntaktische Kompaktheit führt zur schnellen Erfassung der Inhaltsstruktur, hat jedoch komplexe, schwer nachvollziehbare semantische Strukturen zur Folge, was vom Rezipienten entsprechende Aufmerksamkeit und erhöhte Konzentration verlangt.

\section{Der Abstraktionsgrad}

Aufgrund der obigen Untersuchungsergebnisse lässt sich feststellen, dass das Gesetz durch einen hohen Abstraktionsgrad gekennzeichnet ist. Die Abstraktheit liegt schon in seiner Natur oder besser Funktion begründet: Das Gesetz legt abstraktgenerelle Rechtsnormen fest, die dann auf eine Reihe konkreter Fälle angewendet werden. Es normiert und muss deshalb möglichst allgemein d.h. abstrakt sein. Außerdem zeigen sich auf der sprachlichen Ebene des Gesetzestextes zahlreiche abstrakte Formulierungen, hermetische Konstruktionen, was ihn schwer verständlich und nicht selten schwer erklärlich macht. Nun stellt sich die Frage, wo eigentlich die meisten Verständnisschwierigkeiten des Gesetzestextes liegen. Ergeben sich die Dekodierungsprobleme aus den sprachlichen Formulierungen oder beruhen sie auf der "Unkenntnis des modus recipiendi" (Warnke 1996, 212)? In der Sprachwissenschaft werden zwei verschiedene Thesen vertreten: die Stil- und die Wissensthese. Die Erste vertritt die Meinung, dass die Unverständlichkeit von Gesetzen in ihrer sprachlichen Beschaffenheit wurzelt. Dagegen sieht die Andere die Ursachen in der mangelnden Referenz auf die betreffenden Bezugssysteme der Rezipienten.

An dieser Stelle soll noch einmal auf die Querverweise innerhalb des Gesetzestextes hingewiesen werden. Es wurde festgestellt, dass das Gesetz zahlreiche implizite und explizite Verweise enthält. Diese beziehen sich auf andere Rechtsnormen desselben Gesetzes, einige von ihnen sogar auf Rechtsnormen anderer Gesetze, und erst ihr Zusammenspiel ergibt den eigentlichen Sinn. Für die Rezeption bzw. das Dekodieren eines Gesetzestextes reichen die Sprachkenntnisse der Alltagssprache nicht, dafür sind wohl komplexe und genaue Fachkenntnisse unabdingbar - die Kenntnisse also, die nur ausgebildete Juristen besitzen. Deshalb kann das Wort "bürgerlich" im Namen "Bürgerliches Gesetzbuch" mit Sicherheit nicht ein Präsignal an den Adressatenkreis sein. Die Textverfasser haben dabei "so viel alltagsfremdes Wissen implizit vorausgesetzt..., dass ein Verständnis durch Laien gar nicht mehr möglich ist" (Busse 1992, 65). "Bürgerlich" kennzeichnet den Bereich, der durch das BGB geregelt wird: nämlich das bürgerliche Recht.

Um meine Überlegungen abzurunden bzw. kurz zusammenzufassen: Das Verstehen oder Nichtverstehen des Gesetzestextes wird nicht durch seine sprachliche Formu- 
lierung bedingt, sondern ist von dem Vorhandensein, von der "Bereitstellung eines Wissensnetzes" (Warnke 1996, 212) abhängig. Der hohe Abstraktionsgrad des Gesetzes liegt dementsprechend hauptsächlich in seiner komplizierten semantischen Struktur begründet. Abschließend sei noch einmal an das in den einleitenden Gedanken dargestellte Beispiel ( $§ 164,2$ BGB) erinnert - ein guter Beleg dafür, dass die Verständnisschwierigkeiten tatsächlich oft in der Unkenntnis des modus recipiendi wurzeln.

\section{Quellen}

$B G B$ (1996). Frankfurt am Main: Suhrkamp.

BöHME, Dieter (1993):

Formularsammlung für Rechtsprechung und Verwaltung. München: C. H. Beck.

HALTER, Peter (1996):

Entscheidungen des Bundesgerichtshofes in Zivil- und Strafsachen. BGHZ auf CD-ROM, BGHST auf CD-ROM. Handbuch mit Demo-CD. Köln, Berlin, Bonn, München: Carl Heymanns Verlag.

HoPT, Klaus J. (1995):

Vetrags- und Formularbuch zum Handels-, Gesellschafts-, Bank- und Transportrecht. München: C. H. Beck.

Mein Recht im Alltag. Der universelle Helfer bei allen Rechtsproblemen.

NJW ${ }^{9}$ 1970. 1277-1278.; 1976. 329-339.; 1974. 1191-1194.

Predpisi o obligacijskih razmerjih. Prvi zvezek. Zakon o obligacijskih razmerjih s stvarnim kazalom. (1978). Ljubljana: Center za samoupravno normativno dejavnost.

REBMANN, Kurt/SÄCKER, Franz Jürgen (Hg.) (1993): Münchener Kommentar zum Bürgerlichen Gesetzbuch. Band 1. 3. Aufl. München: C. H. Beck.

Zivilrecht. Von ABGB bis WucherG. (1994). 3. Aufl. Wien: Manz.

\section{Literatur}

DE BEAUGRANDE, Robert-Alain / DREsSLER, Wolfgang Ulrich (1981):

Einführung in die Textlinguistik. Tübingen: Max Niemeyer Verlag.

BIERWISCH, Manfred (1992):

Recht linguistisch gesehen. In: Grewendorf, Günther (Hg.): Rechtskultur als Sprachkultur. Frankfurt am Main: Suhrkamp. 42-68.

BRINKER, Klaus (1988):

Linguistische Textanalyse. Eine Einführung in Grundbegriffe und Methoden. 2. Aufl. Berlin: Erich Schmidt Verlag.

BuHLMANn, Rosemarie / FEARNS, Anneliese (1991):

Handbuch des Fachsprachenunterrichts. Unter besonderer Berücksichtigung naturwissenschaftlichtechnischer Fachsprachen. 5. Aufl. Berlin, München, Wien, Zürich, New York: Langenscheidt.

BUSSE, Dietrich (1992):

Recht als Text. Linguistische Untersuchungen zur Arbeit mit Sprachen in einer gesellschaftlichen Institution. Tübingen: Max Niemeyer Verlag.

BuBMANN, Hadumod (1990):

Lexikon der Sprachwissenschaft. 2. Aufl. Stuttgart: Alfred Kröner Verlag.

CANARIS, Claus-Wilhelm / LARENZ, Karl (1995):

Methodenlehre der Rechtswissenschaft. 3. Aufl. Berlin (etc.): Springer-Lehrbuch.

FLUCK, Hans-Rüdiger (1980):

Fachsprachen. Einführung und Bibliographie. München: Francke Verlag.

GrossE, Siegfried (1983):

Syntaktische Besonderheiten der Rechts- und Verwaltungssprache. In: Askedal, John Ole (et al.) (Hg.):

Festschrift für Laurits Salveit. Oslo, Bergen, Tromsö: Universitetsforlaget. 97-109. 
GRUNTAR JERMOL, Ada (1999):

Formale und semantische Strukturen in der deutschen Rechtssprache. Phil. Diss. - Ljubljana.

HAHN, von Walther (1983):

Fachkommunikation. Entwicklung - Linguistische Konzepte - Betriebliche Beispiele. Berlin, New

York: Walter de Gruyter.

HEINEMANN, Wolfgang / VIEHWEGER, Dieter (1991):

Textlinguistik. Eine Einführung. Tübingen: Max Niemeyer Verlag.

HoFFMANN, Lothar (1984):

Kommunikationsmittel Fachsprache. Eine Einführung. 2. Aufl. Berlin: Akademie Verlag.

JOISTEN, Christa (1985):

Sprachkultur der Gesetzessprache. In: Der Deutschunterricht 37 (1985) 1. 47-54.

LEWANDOWSKu, Theodor (1990):

Linguistisches Wörterbuch. 5. Aufl. Heidelberg, Wiesbaden: Quelle \& Meyer.

LinKe, Angelika / Nussbaumer, Markus / PORTMANN, Paul R. (1996):

Studienbuch Linguistik. Tübingen: Max Niemeyer Verlag.

MOHN, Dieter / PELKA, Roland (1984):

Fachsprachen. Eine Einführung. Tübingen: Max Niemeyer Verlag.

NORD, Christiane (1991):

Textanalyse und Übersetzen. Theoretische Grundlagen, Methode und didaktische Anwendung einer übersetzungsrelevanten Textanalyse. 2. Aufl. Heidelberg: Julius Groos Verlag.

OKSAAR, Els (1988):

Fachsprachliche Dimensionen. Tübingen.

SANDRINI, Peter (1996):

Terminologiearbeit im Recht. Deskriptiver begriffsorientierter Ansatz vom Standpunkt des Übersetzers. Wien: Internat.

VATER, Heinz (1996):

Einführung in die Sprachwissenschaft. 2. Aufl. München: Wilhelm Fink Verlag.

VERONESI, Daniela (1998):

Rechtstexte im Vergleich. Das Beispiel von deutschen und italienischen universitären Lehrbüchern. In: Linguistica XXXVIII, 1. Besedilne vrste v medkulturni komunikaciji. - Textsorten in der interkulturellen Kommunikation. Ur. Orešnik, Janez (et al.). Ljubljana: Oddelek za germanske jezike in književnosti Filozofske fakultete Univerze v Ljubljani. 101-123.

WARNKE, Ingo (1996):

Der Wille, in fremdem Namen zu handeln - Vertikalität von Produktions- und Rezeptionsnormen der legistischen Vertextung am Beispiel des $§ 164$ (2) BGB. In: Germanistisches Jahrbuch Ostrava Erfurt für Literaturwissenschafi, Linguistik und Didaktik. OU ? 161/1996. 211-226.

WICHTER, Sigurd (1994):

Experten- und Laienwortschätze. Umriß einer Lexikologie der Vertikalität. Tübingen: Max Niemeyer Verlag. 


\section{Povzetek \\ ALI ZAKONE (ŠE VEDNO) OBEŠAMO PREVISOKO?}

Nemalokrat je slišati kritike, kako nerazumljiv in zapleten je jezik zakonskih besedil. Ker so $\mathrm{v}$ nekaterih zakonih opredeljene pravice in dolžnosti slehernega državljana oziroma civilnih oseb, bi pričakovali, da so pisani v jeziku, ki je razumljiv tudi laikom, ne le pravnim strokovnjakom. Pa ob branju vedno znova ugotovimo, da temu ni tako.

$V$ jezikoslovju lahko zasledimo dve tezi: nekateri trdijo, da so zakoni nerazumljivi in laikom nedostopni predvsem zato, ker so pisani v jeziku, polnem dolgih in zapletenih konstrukcij (denimo nominalni stil, konstrukcije s številnimi desnimi prilastki in podobno). Drugi spet menijo, da slogovna nedovršenost še zdaleč ni glavni vir težav. Do težav pri recepciji zakonskih besedil naj bi prihajalo predvsem zato, ker laiki nimajo ustrezne strokovne podlage, na katero bi »nizali« informacije iz besedila in jih tako znali ustrezno dekodirati.

$\mathrm{V}$ članku želim na podlagi besedilne analize dokazati, katero od obeh tez je mogoče potrditi. Korpus besedil, ki zaradi boljše primerljivosti in utemeljevanja poleg zakona vključuje še nekatere druge pravne besedilne vrste, sem analizirala po naslednjih parametrih: situacijski tip, funkcija, tematska progresija, morfološko-sintaktične značilnosti, besedišče in stopnja abstraktnosti.

Rezultati raziskave so pokazali, da zakonsko besedilo vsebuje nemalo okornih konstrukcij, ki bi jih bilo pogosto mogoče razvezati v preprostejše in bralcu prijaznejše. Poleg tega težave pri recepciji povzročajo številni termini, ki se na formalni ravni sicer ujemajo $z$ nestrokovnimi besedami, a so vsebinsko drugače definirani. Zakon marsikateri termin sicer definira, vendar so te vsebinske razlage podane zelo abstraktno.

Še posebej zanimivi so rezultati raziskave o koherentnosti in abstraktnosti zakonskega besedila. Paragrafi (osnovne enote zakona), ki tvorijo posamezna poglavja (naslove/odseke) delujejo kot bolj ali manj zaključene celote. Čeprav tematsko sovpadajo, vendarle ne bi mogli reči, da - vsaj za laika - predstavljajo koherentno besedilo. Še posebej zapletena so zakonska besedila zato, ker nas zakonodajalec $v$ posameznih paragrafih za dodatno razlago ali restrikcijo norme napoti na druge paragrafe istega, včasih celo katerega drugega zakona oziroma zakonika. Ker zakoni določajo norme, te pa so vedno okvirne narave, morajo biti pisani dovolj široko in zato abstraktno. Ponazoritev na konkretnih primerih bi bila seveda mnogo bolj razumljiva, a bi zakon s tem izgubil svojo normativno funkcijo. Če na kratko povzamem: za razumevanje zakonskih besedil je potrebno predvsem ustrezno strokovno znanje. Zapletena jezikovna struktura sicer otežuje recepcijo besedila, a je pri tem očitno bolj postranskega pomena. 